\title{
Apoptotic cell instillation after bleomycin attenuates lung injury through hepatocyte growth factor induction
}

\author{
Ye-Ji Lee*, Changsuk Moon*, Seung Hae Lee*, Hyun-Jeong Park*, Ju-Young Seoh*, \\ Min-Sun Cho" and Jihee Lee Kang*
}

ABSTRACT: Apoptotic cell clearance by macrophages and neighbouring tissue cells induces hepatocyte growth factor (HGF) secretion. HGF plays a key role in alveolar epithelial regeneration and reconstruction after lung injury. Direct in vivo exposure to apoptotic cells enhances HGF production, resulting in attenuation of pulmonary injury.

We investigated the direct effect of in vivo exposure to apoptotic cells in bleomycin-stimulated lungs (2 days old) on HGF induction. Furthermore, sequential changes of bleomycin-induced HGF production following apoptotic cell instillation related to the changes in inflammatory and fibrotic responses were assessed.

At $2 \mathrm{~h}$ after apoptotic cell instillation into bleomycin-stimulated lungs, the levels of HGF mRNA and protein production, and apoptotic cell clearance by alveolar macrophages were enhanced. Furthermore, HGF induction persistently increased following apoptotic cell instillation up to 21 days after bleomycin treatment. Apoptotic cell instillation attenuated bleomycin-induced proinflammatory mediator production, inflammatory cell recruitment and total protein levels. Apoptotic cell instillation also induced antiapoptotic and antifibrotic effects. These antiinflammatory and antiapoptotic effects could be reversed by co-administration of HGFneutralising antibody.

These findings indicate that in vivo exposure to apoptotic cells enhances transcriptional HGF production in bleomycin-stimulated lungs, resulting in attenuation of lung injury and fibrosis.

KEYWORDS: Alveolar macrophages, apoptotic cell clearance, lung fibrosis, lung inflammation

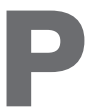

ulmonary fibrosis is an inflammatory interstitial lung disease characterised by an accumulation of alveolar macrophages and neutrophils in the lower respiratory tract, alveolar epithelial injury, and aberrant alveolar wound repair [1, 2]. Previous studies have indicated that efficient alveolar epithelial cell repair is critical for normal healing without fibrosis [3-5]. Because apoptotic cell clearance results in the release of growth factors and the activation of signalling molecules involved with the maintenance of the epithelium and endothelium, VANDIVIER et al. [6] proposed that alveolar integrity maintenance is critically dependent on intact mechanisms of apoptotic cell clearance. Recognition and ingestion of apoptotic neutrophils by alveolar macrophages in vitro and in vivo during bacterial pneumonia induces higher levels of hepatocyte growth factor (HGF) production [7]. The importance of this response is probably due to the well-defined role of HGF as a growth factor for alveolar type II and bronchial epithelial cells. In this paradigm, following tissue injury, apoptotic cell clearance may be associated with restoration of normal structural cells.

There is now ample evidence that HGF regulates such diverse cellular processes as cell survival, proliferation, migration and differentiation in various organs [8-12]. In lungs, HGF is produced by mesenchymal and bronchial epithelial cells, and alveolar macrophages [13]. Recently, WATANABE et al. [14] reported that increased endogenous HGF in mouse lungs following HGF gene transfection decreased pro-inflammatory cytokine levels and collagen synthesis after bleomycin injury.

For editorial comments see page 289.

This article has supplementary material available from www.erj.ersjournals.com/site/misc/statements.xhtml

AFFILIATIONS

*Dept of Physiology, Tissue Injury Defense Research Center, School of Medicine, Ewha Womans University, \#Dept of Microbiology, School of Medicine, Ewha Womans University, and

'Dept of Pathology, School of Medicine, Ewha Womans University, Seoul, Korea.

CORRESPONDENCE

J.L. Kang

Dept of Physiology, Tissue Injury

Defense Research Center, School of

Medicine

Ewha Womans University

911-1 Mok-6-dong

Yangcheon-gu

Seoul 158-056

Korea

E-mail: jihee@ewha.ac.kr

Received:

June 062011

Accepted after revision:

Dec 282011

First published online:

March 222012 
Additionally, HGF antagonises the profibrotic actions of transforming growth factor (TGF)- $\beta 1$ by intercepting Smad signal transduction through diverse mechanisms $[15,16]$.

In the present study, we examined the relationship between recognition and clearance of apoptotic cells and successful tissue repair during lung inflammation proceeding to fibrosis. To explore this connection, apoptotic cells were directly instilled into murine lungs at a very early phase after bleomycin treatment. We focused on the direct in vivo effects of apoptotic cell instillation into the bleomycin-stimulated lungs on HGF mRNA and protein expression. We also investigated sequential changes of bleomycin-induced HGF production following apoptotic cell instillation related to the changes in inflammatory and fibrotic responses.

\section{MATERIALS AND METHODS}

Further details of the materials and methods may be found in the online supplementary material.

\section{Animal protocols}

Specific pathogen free, male C57BL/6 mice (Orient Bio, Sungnam, Korea) weighing 20-22 g were used in all experiments. The Animal Care Committee of the Ewha Medical Research Institute (Seoul, Korea) approved the experimental protocol.

Mouse pharyngeal aspiration was used for administration of the test solution [17]. Briefly, animals were anaesthetised with a mixture of ketamine and xylazine (45 and $8 \mathrm{mg} \cdot \mathrm{kg}^{-1}$ intraperitoneally, respectively), and placed individually on a board in a near-vertical position. The animal's tongue was extended with lined forceps. The test solution $(30 \mu \mathrm{L})$ containing bleomycin ( $5 \mathrm{U} \cdot \mathrm{kg}^{-1}$ body weight) or sterile saline $(0.9 \%$ sodium chloride) was then placed posteriorly in the throat and aspirated into the lungs. 2 days after bleomycin stimulation, saline alone or $10 \times 10^{6}$ apoptotic or viable Jurkat cells in $50 \mu \mathrm{L}$ saline were administered intratracheally through pharyngeal aspiration [17, 18] and mice were euthanised $2 \mathrm{~h}$ after apoptotic cell instillation, and on days 3, 5, 7, 14 and 21 following bleomycin treatment.

For anti-HGF antibody inhibition experiments, $250 \mu \mathrm{g}$ of either neutralising rabbit polyclonal antibody to mouse HGF or normal goat immunoglobulin (Ig)G (Santa Cruz Biotechnology, Santa Cruz, CA, USA) in $200 \mu \mathrm{L}$ saline was administrated i.p. at the same time with i.t. instillation of $10 \times 10^{6}$ apoptotic Jurkat $\mathrm{T}$-cells in $50 \mu \mathrm{L}$ saline or $50 \mu \mathrm{L}$ saline alone into the bleomycinstimulated lungs (2 days). Sequentially, the antibody was administrated once more at 5 days after bleomycin treatment. Mice were euthanised on days 3 or 7 following bleomycin treatment.

Bronchoalveolar lavage cells, lung tissue and cell counts

Bronchoalveolar lavage (BAL) was performed and cell counts were determined using an electronic Coulter Counter fitted with a cell-sizing analyser (Coulter Model ZBI with a Channelizer 256; Coulter Electronics, High Wycombe, UK). BAL cells were isolated and cytospins were made to assess phagocytic indexes $[19,20]$.

\section{Induction of apoptosis}

Apoptosis of Jurkat T-cells was induced by ultraviolet irradiation, as previously described [21].

\section{Alveolar macrophage culture}

Suspended alveolar macrophages from mice were $>95 \%$ viable, as determined by trypan blue dye exclusion. Alveolar macrophages were isolated by adhesion $(60 \mathrm{~min})$ and cultured in serum-free X-VIVO medium (Lonza, Basel, Switzerland).

\section{In vitro exposure of macrophages to stimulants for cytokine analysis}

Murine RAW 264.7 macrophages $\left(10^{6} \mathrm{cells} \cdot \mathrm{mL}^{-1}\right)$ were stimulated with apoptotic or viable Jurkat T-cells $\left(3 \times 10^{6}\right.$ cells $\left.\cdot \mathrm{mL}^{-1}\right)$ and the supernatants were collected $18 \mathrm{~h}$ later. In some experiments, lipopolysaccharide (LPS) $\left(1 \mathrm{ng} \cdot \mathrm{mL}^{-1}\right)$ or bleomycin $\left(5 \mu \mathrm{g} \cdot \mathrm{mL}^{-1}\right)$ was added to stimulate cytokine production. Apoptotic or viable cells, or apoptotic cells and anti-HGF receptor (HGFR) (c-Met) antibody $\left(10 \mu \mathrm{g} \cdot \mathrm{mL}^{-1}\right)$ were added at the same time of the stimulus, and supernatants were collected 18 h later.

\section{ELISA}

HGF, TGF- $\beta 1$, tumour necrosis factor (TNF)- $\alpha$ and macrophage inflammatory protein (MIP)-2 were measured using ELISA kits as per the manufacturer's instructions.

\section{RT-PCR}

Total RNA was isolated from lung tissue using TRIzol ${ }_{\mathbb{R}}$ reagent (Life Technologies $^{\mathrm{TM}}$, Grand Island, NY, USA) according the manufacturer's instructions. HGF mRNA levels were determined using relative quantitative RT-PCR kits.

\section{Measurement of total protein}

Protein concentrations of the BAL samples were measured according to the method of HARTREE [22].

\section{Lung histology}

Lung tissue was fixed with $10 \%$ buffered formalin and embedded in paraffin. 3- $\mu \mathrm{m}$ sections were stained with haematoxylin-eosin (H\&E) or Masson's trichrome to evaluate inflammation or collagen deposition, respectively.

\section{Immunohistochemistry for HGF}

Paraffin-embedded lung tissues were immunostained for HGF (rabbit polyclonal anti-HGF; Santa Cruz Biotechnology).

\section{DNA damage and apoptosis in lung tissue}

DNA fragmentation of apoptotic cells was detected in the lung tissue using a terminal deoxynucleotidyltransferase-mediated deoxyuridine triphosphate nick end-labelling (TUNEL) kit (Promega, Madison, WI, USA), according to the manufacture's protocols.

\section{Western blot analysis}

Lung tissue homogenate samples were separated on $10 \%$ sodium dodecylsulfate-polyacrylamide gels. Separated proteins were electrophoretically transferred onto nitrocellulose paper. The membranes were probed with specific antibodies to cleaved caspase-3, proliferating cell nuclear antigen, $\alpha$-tubulin or $\beta$-actin antibodies and visualised by chemiluminescence. 


\section{Caspase-3 and -9 activities}

The bioactivity of caspase-3 and -9 was measured with a Fluorometric Assay Kit (ABcam, Cambridge, UK). In brief, lung homogenate samples were incubated with the caspase-3 substrate DEVD-AFC or the caspase-9 substrate LEHD-AFC. The fluorescence of the cleaved substrates was determined at an excitation wavelength of $400 \mathrm{~nm}$ and an emission wave length of $505 \mathrm{~nm}$.

\section{Measurement of hydroxyproline}

Lung hydroxyproline content was measured using a hydroxyproline assay kit (Nanjing Jiancheng Bioengineering Institute, Nanjing, China) according to the manufacturer's instructions.

\section{Statistical analysis}

Data are presented as mean \pm SEM. ANOVA was applied for multiple comparisons and Tukey's post hoc test was applied where appropriate. Unpaired t-tests were used for comparisons of two sample means. A p-value of $<0.05$ was considered statistically significant. All data were analysed using JMP software (SAS Institute, Cary, NC, USA).

\section{RESULTS}

\section{Effects of in vivo instillation of apoptotic cells into bleomycin-stimulated lungs on HGF production and apoptotic cell clearance}

To examine the direct effect of in vivo instillation of apoptotic cells on HGF production in bleomycin-stimulated lungs, apoptotic or viable Jurkat cells, or saline were instilled i.t. 2 days after bleomycin treatment. $2 \mathrm{~h}$ after in vivo instillation of apoptotic cells into inflamed lungs, nearly all apoptotic cells were cleared and apoptotic fragments were apparent in macrophages [18]. Our previous in vitro study demonstrated that apoptotic cell-induced HGF mRNA expression in macrophages peaked at $2 \mathrm{~h}$ [23]. Thus, BAL fluid and lung tissue were obtained $2 \mathrm{~h}$ after cell instillation to analyse HGF production and mRNA expression. The levels of HGF in BAL fluid were elevated in the bleomycin plus saline (BLM+Sal) group as compared with the saline-alone control group (fig. 1a). The instillation of apoptotic cells further enhanced HGF production over that of the BLM+Sal group, while instillation of viable cells did not alter baseline HGF production. To determine the production of $\mathrm{HGF}$ at the mRNA level, relative quantitative RT-PCR was performed using mRNA extracted from total lung tissue. As seen for HGF protein production, HGF mRNA levels were further enhanced by in vivo instillation of apoptotic cells, but not by viable cells (fig. $1 \mathrm{~b}$ ). At $2 \mathrm{~h}$, in vivo exposure to apoptotic cells also enhanced HGF production in cultured alveolar macrophages as compared with HGF production by alveolar macrophages following exposure to saline or viable cells (fig. 1c).

The clearance of apoptotic Jurkat cells by alveolar macrophages $2 \mathrm{~h}$ after instillation of apoptotic cells into bleomycinstimulated lungs was robust, when compared with saline or viable cells (fig. 1d). In contrast, HGF production and mRNA levels, and phagocytic indexes in alveolar macrophages from naïve lungs were not significantly changed by apoptotic cell instillation (fig. S1).

An additional question was whether the effect of apoptotic cells was restricted to T-cells or whether other apoptotic cells would have the same effect. Therefore, apoptotic HeLa epithelial cells were instilled into the bleomycin-stimulated lungs, and the effects on HGF mRNA expression and protein levels in lungs and cultured alveolar macrophages were measured. There was no significant difference between the effects of the Jurkat T-cells compared with the HeLa cells. Apoptotic HeLa cell instillation also induced increases in HGF mRNA and protein expression as well as clearance ability of apoptotic cells by alveolar macrophages (fig. S2).

\section{Serial changes in HGF production and $m R$ RA expression}

To further detect serial changes in the levels of HGF in BAL fluid after instillation of apoptotic cells, BAL fluid was examined 321 days after bleomycin treatment. HGF protein levels gradually increased up to 21 days after bleomycin treatment (fig. 2a). The instillation of apoptotic cells further enhanced the HGF protein levels each day after bleomycin treatment.

Similarly, HGF mRNA expression in lung tissue following bleomycin treatment gradually increased up to day 21 (fig. 2b). As seen for the HGF protein levels, HGF mRNA levels were further enhanced following instillation of apoptotic cells, reaching peak levels at day 21 (fig. $2 b$ ). Since HGF protein and mRNA expression followed similar kinetics after bleomycin treatment and apoptotic cell instillation, HGF production in this context is probably regulated at the transcriptional level.

\section{Cellular source of HGF in lung tissue}

To determine the cellular source of HGF in the lung, an immunohistochemical study was performed on lung tissue using an anti-HGF polyclonal antibody. HGF expression was mainly localised to alveolar macrophages and, to some extent, alveolar epithelial cells at days 3-21 after bleomycin treatment with saline (fig. $2 \mathrm{~d}-\mathrm{f}$ ) or apoptotic cell instillation (fig. $2 \mathrm{~h}-\mathrm{j}$ ) (data not shown at days 3 and 14). In contrast, staining for HGF was very weak in sections of unstimulated saline controls (fig. 2c). In addition to these cell types, positive staining for HGF was also shown in mesenchymal cells of the lung from these experimental groups at 21 days (fig. $2 \mathrm{f}$ and $\mathrm{j}$ ). Interestingly, there was strong expression of HGF in alveolar macrophages following the instillation of apoptotic cells at 21 days (fig. 2j). No specific staining for HGF was observed in sections of lungs at 7 days after bleomycin treatment that had been incubated with IgG isotype control (fig. 2g).

\section{Changes in phagocytic indexes in alveolar macrophages}

To determine whether the ability of alveolar macrophages to clear endogenous apoptotic cells is affected by apoptotic cell instillation, phagocytic indexes were measured in BAL alveolar macrophages 3-21 days after bleomycin treatment. The phagocytic indexes in alveolar macrophages from bleomycin-stimulated lungs were higher than the phagocytic indexes of the saline controls at each day after bleomycin treatment (fig. 2k). Furthermore, the phagocytic indexes in alveolar macrophages from the BLM plus apoptotic Jurkat cells (BLM+ApoJ) group were significantly higher compared with the BLM+Sal group at the same time-points.

\section{Reduction in inflammatory responses following instillation of apoptotic cells into bleomycin-stimulated lungs} TNF- $\alpha$ and MIP-2 protein levels did not vary in bleomycinstimulated lungs lavaged $2 \mathrm{~h}$ after instillation of saline, 


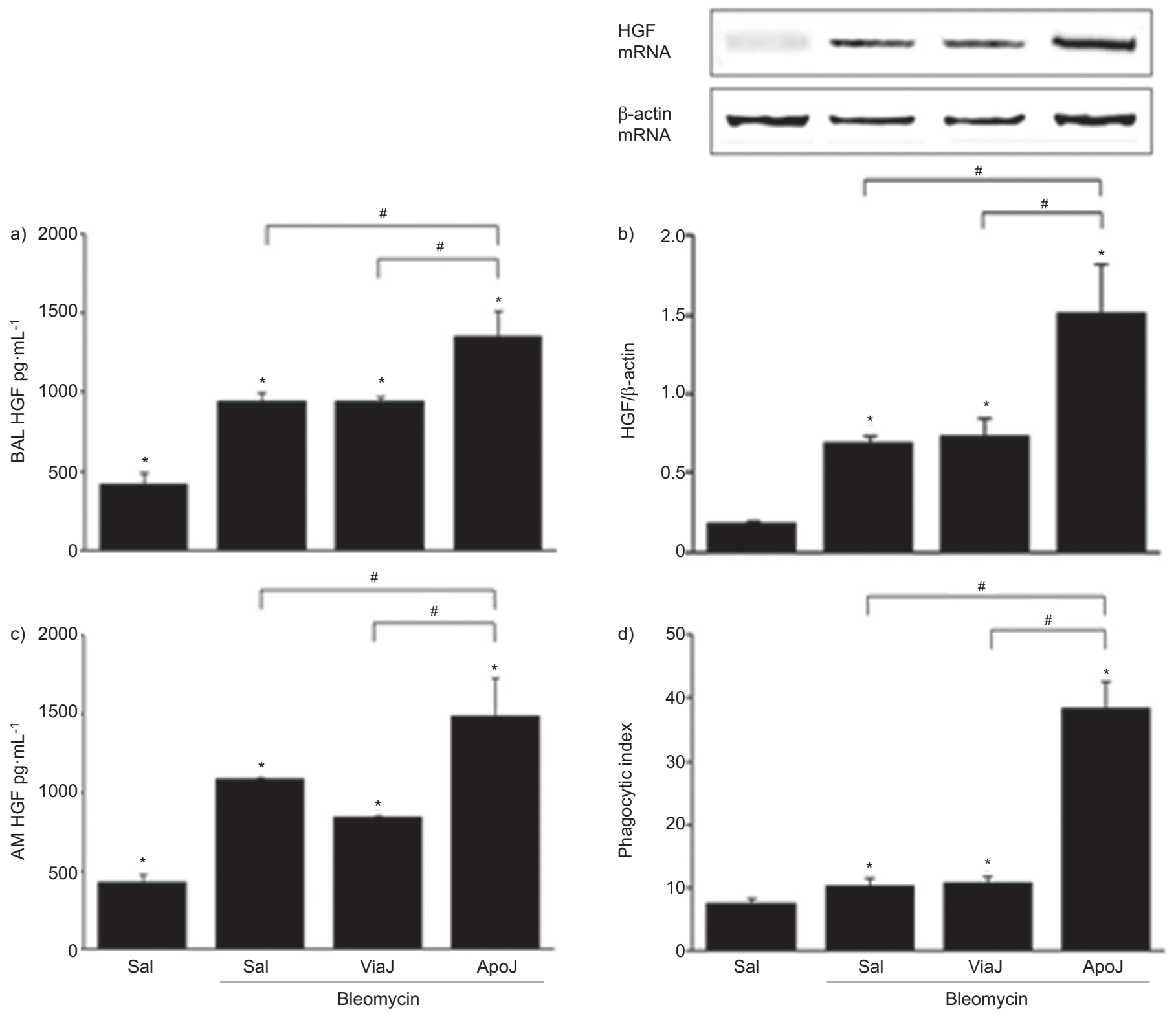

FIGURE 1. In vivo production and mRNA expression of hepatocyte growth factor (HGF) in bleomycin-stimulated lungs are increased by apoptotic cell clearance. Bronchoalveolar lavage (BAL) was performed $2 \mathrm{~h}$ after intratracheal instillation of saline (Sal) alone, or bleomycin plus Sal, apoptotic Jurkat T-cells (ApoJ) or viable Jurkat T-cells (ViaJ). a) HGF production in BAL fluid samples was quantified by ELISA. b) HGF mRNA levels in lung homogenates were analysed by relative quantitative RT-PCR and normalised to $\beta$-actin mRNA levels. c) HGF production in alveolar macrophage (AM) cultures was quantified by ELISA. d) Phagocytic indexes were measured in BAL AMs. Data are presented as mean \pm SEM. $n=5-10$ mice per group. *: $p<0.05$ compared with Sal alone; ${ }^{*}: p<0.05$ between groups.

apoptotic cells or viable Jurkat T-cells (data not shown). However, there was a significant reduction of $\mathrm{TNF}-\alpha$ and MIP-2 in the BAL fluid of the BAL+ApoJ group at 3 days postbleomycin treatment as compared with the BLM+Sal group (fig. $3 a$ and $b$ ). The TNF- $\alpha$ and MIP-2 levels in all groups rapidly declined thereafter, and the differences were not significant 7-21 days after bleomycin treatment.

With HGF induction, bleomycin treatment increased TGF- $\beta 1$ expression in BAL fluid as compared with the saline control BAL fluid 2 days after bleomycin treatment (fig. 3c). The levels of TGF- $\beta 1$ declined by days $3-5$ and increased again by day 21 with a second peak (fig. $3 \mathrm{~d}$ ). TGF- $\beta 1$ levels in the
BLM+ApoJ group were greater at days 2 and 3, and lower at days 14 and 21 compared with those in the BLM+Sal group, respectively. These data suggest that in vivo instillation of apoptotic cells results in upregulation of bleomycin-induced TGF- $\beta 1$ during the early phase, but down-regulation of TGF$\beta 1$ at the later phase.

Neutrophil recruitment into the lungs occurred within 3 days, peaked after 7 days and declined by day 21 post-bleomycin treatment (fig. 3e). Alveolar macrophage numbers peaked after 14 days and slightly declined by 21 days after bleomycin treatment (fig. 3f). BAL total protein levels progressively increased and peaked at day 14 before slightly declining by 


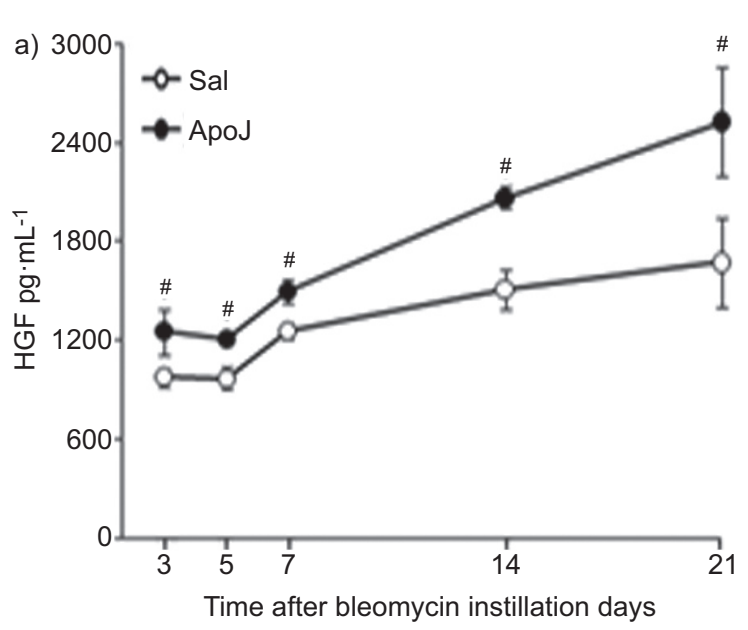

b)
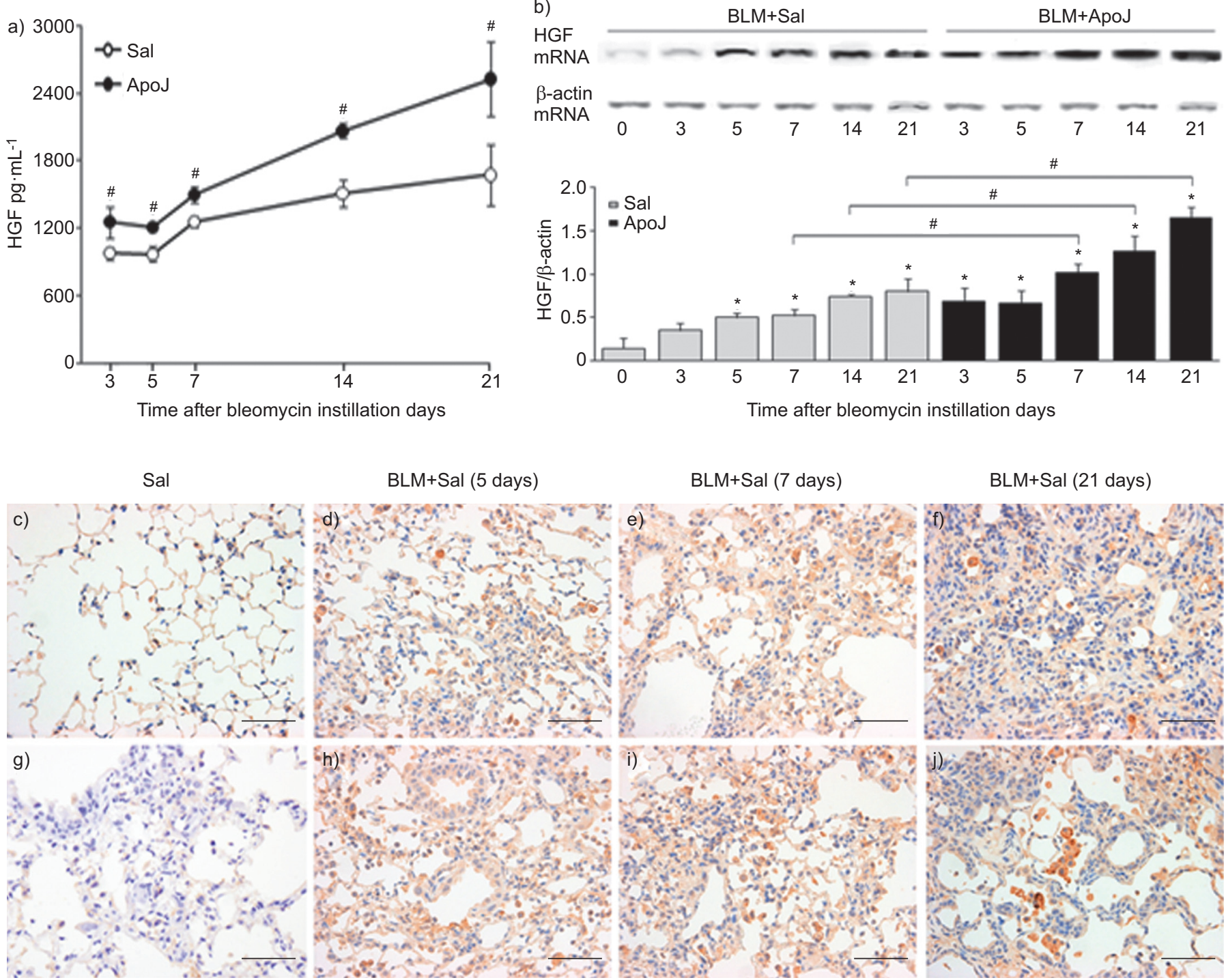

$\mathrm{BLM}+\mathrm{Sal}(7$ days $)$

BLM+ApoJ (5 days)

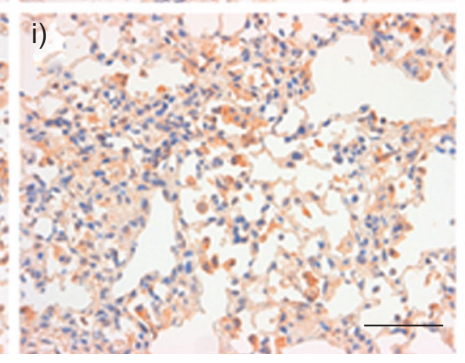

BLM+ApoJ (7 days)

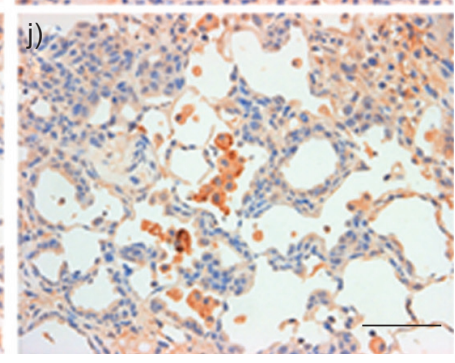

BLM+ApoJ (21 days)

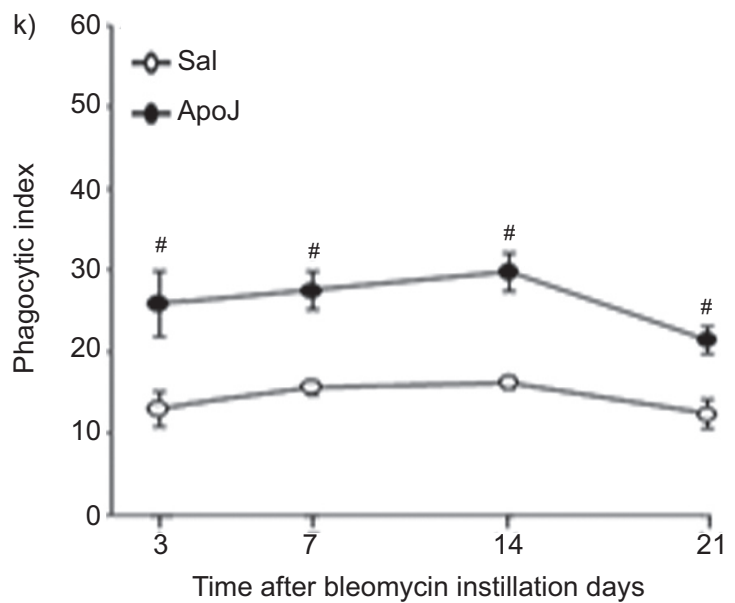

FIGURE 2. Changes in hepatocyte growth factor (HGF) protein levels in bronchoalveolar lavage (BAL) fluid and HGF mRNA expression in lung tissue. Mice were instilled intratracheally with saline (Sal) or apoptotic Jurkat T-cells (ApoJ) on day 2 and euthanised on days 3-21 following bleomycin treatment. a) HGF levels in BAL fluid samples were quantified at each time-point by ELISA. b) HGF mRNA levels in lung homogenates were analysed by relative quantitative RT-PCR and were normalised to $\beta$ actin mRNA levels. $n=5$ mice per group. Immunohistochemical staining of lungs with $c-f$, $\mathrm{h}$-j) anti-HGF antibody or g) immunoglobulin $\mathrm{G}$ isotype control at time-points after bleomycin (BLM) treatment. Representative results from five mice per group are shown. $\mathrm{k}$ ) Changes in apoptotic cell clearance by alveolar macrophages. Phagocytic indexes were measured in BAL alveolar macrophages. Data are presented as mean \pm SEM. $n=5-10$ mice per group. Scale bars $=10 \mu \mathrm{m}$. *: $p<0.05$ compared with Sal control; ${ }^{*}: p<0.05$ for ApoJ versus Sal. 
day 21 (fig. 3g). Instillation of apoptotic cells significantly reduced BAL neutrophil numbers and protein levels 3-21 days and alveolar macrophage numbers 14 and 21 days postbleomycin treatment.

Since the maximal level of neutrophils in BAL fluid was observed on day 7 , sections of lung tissue from these mice at 7 days postbleomycin treatment were $\mathrm{H} \& \mathrm{E}$ stained. Histological findings revealed alveolar thickening due to infiltration of inflammatory cells (fig. 3h-j). Furthermore, endothelial and alveolar barriers were destroyed and invaded by inflammatory cells. The number of inflammatory cells in alveolar spaces and the lung parenchyma decreased, and only mild alveolar structure destruction was present in lung tissue instilled with apoptotic cells as compared to lungs from the BLM+Sal group. Additionally, we assessed DNA damage and apoptosis levels in lungs at day 7 postbleomycin treatment using TUNEL staining. Apoptotic cell instillation reduced the number of TUNEL-positive alveolar epithelial cells (fig. 4a and b). The decrease in apoptosis levels was confirmed by Western blot analysis for the cleaved form of caspase-3 (Asp) (fig. 4c).

We also examined serial changes in cytokines, including HGF, TGF- $\beta 1$, TNF- $\alpha$ and MIP-2, as well as inflammatory responses, including inflammatory cell accumulation and total protein levels after instillation of viable Jurkat cells into the bleomycinstimulated lungs. As expected, there were no statistical differences between the effects on the BLM+ViaJ group compared with the BLM+Sal group at days 3-14 after bleomycin treatment (data not shown). In addition, since all 10 mice treated with viable cells died within 21 days after bleomycin treatment, the effects between two groups could not be compared.

\section{Reduction of lung fibrosis following instillation of apoptotic cells}

Collagen content was assessed by measuring hydroxyproline levels in lung tissue 3-21 days after bleomycin treatment. The hydroxyproline content in lung tissue from the BAL+Sal group increased by day 14 and peaked at day 21 following bleomycin treatment (fig. 5a). However, instillation of apoptotic cells significantly reduced bleomycin-induced hydroxyproline content. Collagen-stained interstitial areas with damaged alveolar structures caused by instillation of apoptotic cells decreased, as compared with lungs from the BLM+Sal group at day 21 postbleomycin treatment (fig. 5b).

\section{Reduction in inflammation and apoptosis following instillation of apoptotic cells into bleomycin-stimulated lungs is mediated by HGF}

To confirm that the reduction of inflammation and apoptosis was mediated by apoptotic cell induction of HGF, anti-HGF neutralising antibody or isotype control antibody was co-administered with the apoptotic Jurkat cells into bleomycin-stimulated lungs (2 days), or administered once more sequentially 5 days after bleomycin treatment. Changes in inflammatory and apoptotic responses were evaluated at days 3 or 7 after bleomycin treatment. The anti-HGF antibody completely reversed the reduction of pro-inflammatory mediators, including TNF- $\alpha$ and MIP-2, neutrophil recruitment, and total protein levels (fig. S3a-e), as well as caspase-3 and -9 activities (fig. S3f and g). The IgG isotype control antibody had no effects. However, the levels of TGF- $\beta 1$ at 3 days after bleomycin treatment showed a trend towards a further increase in response to the anti-HGF antibody, although it was not statistically significant compared with the BLM+ApoJ group (fig. S3c). At 7 days, the anti-HGF antibody did not affect the levels of TGF- $\beta 1$ (data not shown).

\section{Inhibitory effects of endogenously produced HGF on production of pro-inflammatory cytokines by macrophages in vitro}

We examined whether apoptotic cell-induced HGF expression also reduces inflammatory cytokine expression in vitro. Macrophages were treated with either LPS $\left(1 \mathrm{ng} \cdot \mathrm{mL}^{-1}\right)$ or bleomycin $\left(5 \mu \mathrm{g} \cdot \mathrm{mL}^{-1}\right)$ and, at the same time, exposed to apoptotic or viable Jurkat T-cells. As expected, apoptotic cell treatment inhibited LPS-induced production of TNF- $\alpha$ and MIP-2 production, but not of HGF, which was enhanced significantly when compared with macrophages treated with LPS alone and LPS plus viable cells (fig. S4a-c). Similar results were observed for macrophages stimulated with bleomycin (fig. S4a-c). To examine the effects of released HGF on production of pro-inflammatory cytokines, anti-HGFR (c-Met) antibody was added to LPS- or bleomycinstimulated macrophages with apoptotic cells. Treatment with anti-HGFR antibody was found to reverse the suppression of TNF- $\alpha$ and MIP-2 production caused by apoptotic cells in macrophages stimulated with LPS or bleomycin (fig. S4d and e). Taken together with in vivo findings, these results show that HGF produced endogenously in response to apoptotic cell treatment plays a role in inhibiting the generation of inflammatory cytokines in macrophages in vitro.

\section{Changes of survival rate following instillation of apoptotic cells into bleomycin-stimulated lungs}

Of the mice treated with bleomycin, $80 \%$ died within 21 days (fig. 6). However, instillation of apoptotic cells reduced the death rate (14\% mortality).

\section{DISCUSSION}

Recognition and phagocytosis of apoptotic cells by macrophages promote the resolution of acute inflammation through TGF- $\beta$ secretion $[18,24]$. However, unregulated TGF- $\beta$ production can lead to a progressive fibrotic process. Thus, a probable role for apoptotic cell recognition and clearance in fibrotic alterations needs to be investigated. Little is known regarding the effects of apoptotic cell clearance on tissue repair mediated by other growth factors. Recent studies have demonstrated apoptotic cell-induced HGF production, which is a key factor involved in alveolar epithelial regeneration and reconstruction after lung injury [12-14]. Therefore, we investigated whether in vivo apoptotic cell instillation into lungs leads to increased HGF production resulting in attenuation of bleomycin-induced lung inflammation and fibrosis. Our studies were carried out with apoptotic Jurkat T-cells that allowed us to distinguish them from the dominant inflammatory cells (neutrophils and macrophages/monocytes). In vitro studies have shown similarity in the way macrophages recognise and respond to different apoptotic cell types; however, neutrophils that undergo secondary necrosis would introduce additional pro-inflammatory and toxic materials. Furthermore, a variety of cell types, including fibroblasts, macrophages, neutrophils, smooth muscle cells and epithelial cells, could be unaccounted sources of HGF, but Jurkat T-cells neither contain nor produce detectible HGF. Thus, we used Jurkat T-cells for this investigation. 

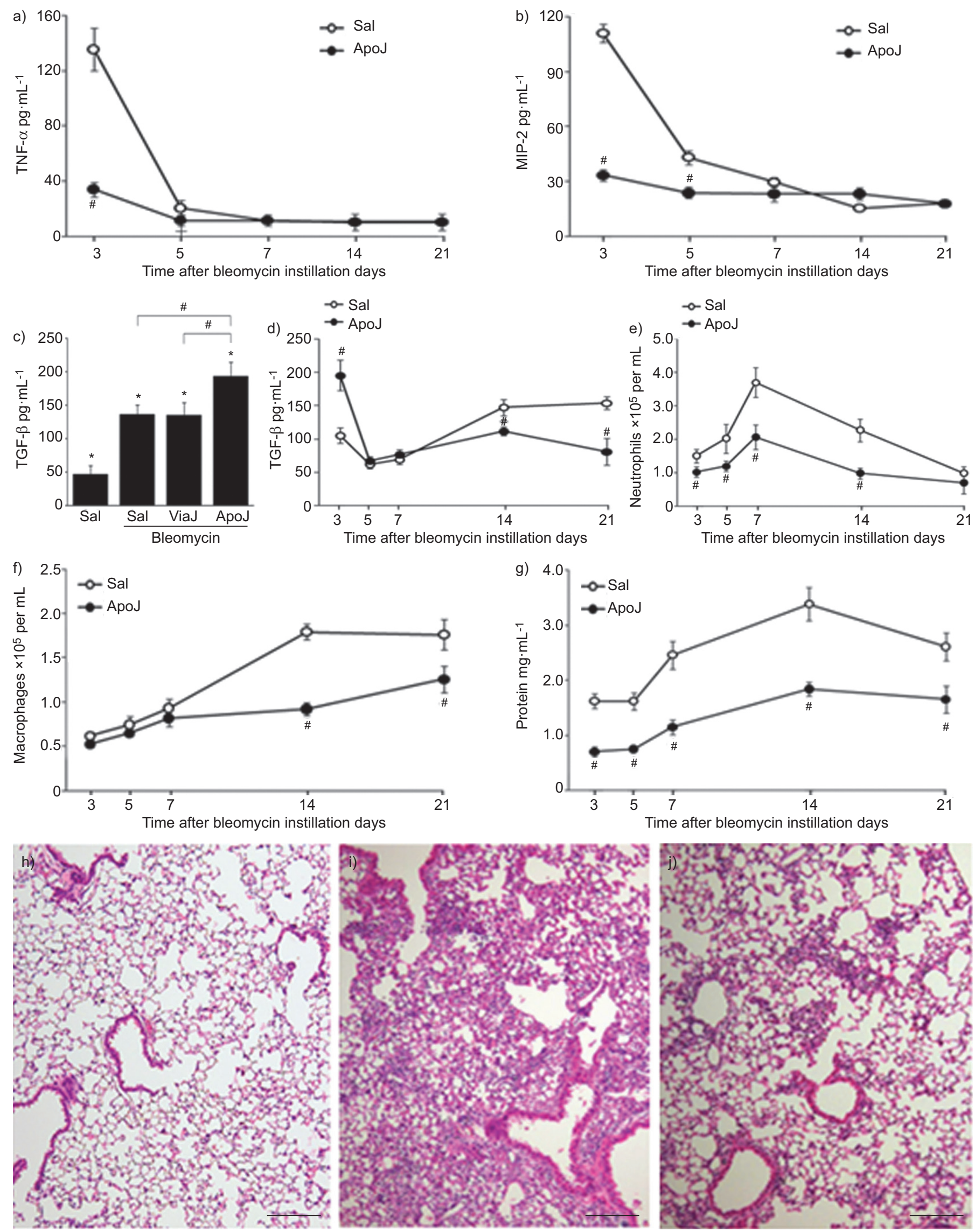


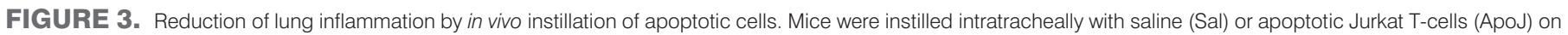

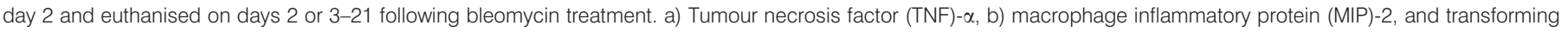

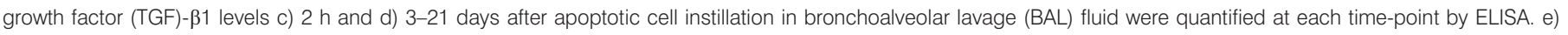

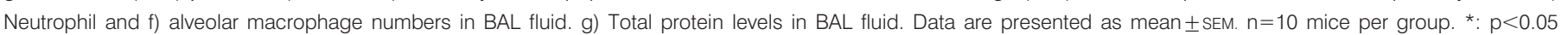

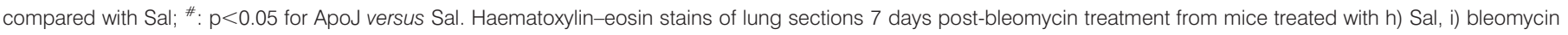
plus Sal and j) bleomycin plus ApoJ. Scale bars $=10 \mu \mathrm{m}$. Representative results from 10 mice per group are shown

We initially focused on the immediate responses after instillation of apoptotic cells into lungs 2 days after bleomycin stimulation to allow for comparisons between viable and apoptotic cells. Here, we demonstrated that in vivo exposure to apoptotic Jurkat cells resulted in further induction of HGF at the mRNA and protein levels. We also demonstrated that HGF expression was induced specifically by apoptotic cell recognition systems as apoptotic, but not viable Jurkat cell-induced HGF expression.

ADAMSON and BAKOWSKA [25] demonstrated that the levels of HGF in BAL fluid increased as early as day 3, peaked at days 7-14 and dropped sharply after day 14 following bleomycin treatment in rats. In our murine model, HGF in BAL fluid increased up to 21 days following bleomycin treatment. HGF mRNA expression in murine lung tissue was induced over a similar time course following bleomycin treatment.

The factors that lead to up-regulation of HGF expression in the setting of bleomycin-induced lung injury are beyond the scope of this project, but a variety of proteases and inflammatory cytokines, such as interleukin (IL)- 6 , IL- $1 \alpha$, IL-1 $\beta$ and TNF- $\alpha$, have been shown to increase HGF mRNA and protein expression [26-30]. Other data [7] provide an additional variable in persistent HGF production that involves previous exposure to endogenous apoptotic cells or cell debris in lung injury lesions since the phagocytic indexes in BAL alveolar macrophages increased following bacterial infections. Here, our data advances these concepts, because it shows that in vivo instillation of apoptotic cells led to further induction of HGF mRNA and protein up to 21 days after bleomycin treatment from the already elevated basal levels due to lung injury. This increased HGF production may be partially due to the increased phagocytic abilities of alveolar macrophages for endogenous apoptotic cells since the increases in phagocytic indexes were maintained in the BLM+ApoJ group as compared with the BLM+Sal group. Finally, additional HGF contribution from tissue cells, particularly the epithelial and mesenchymal cells, may have been affected by direct interaction with apoptotic cells or by autocrine/paracrine induction of HGF [31-33]. Indeed, our immunohistochemical studies indicated that the cellular source of HGF was mainly alveolar macrophages and, to some extent, alveolar epithelial cells and mesenchymal cells (particularly at 21 days after bleomycin treatment) with saline or apoptotic cell instillation.

The mechanisms of the enhanced ability of alveolar macrophages to clear endogenous apoptotic cells are not well understood, but appear to involve a mechanistic link between HGF induction and homeostatic regulation of apoptotic cell clearance. Increased efferocytosis in a time-dependent fashion was also shown in zymosan-induced peritonitis animal models, which was mirrored by peroxisome proliferatoractivated receptor (PPAR) $-\gamma$ expression and activation [34]. PPAR $-\gamma$ and Rac1 are positive regulators of efferocytosis of apoptotic cells [6, 35-37]. Exposure to apoptotic cells has been shown to induce cyclo-oxygenase (COX)-2-derived production of prostaglandins (PGs), including $\mathrm{PGE}_{2}$ and $\mathrm{PGJ}_{2}$, which are endogenous stimulants for Rac1 and PPAR- $\gamma$ activation, respectively $[38,39]$. Based on these findings, our laboratory is actively investigating the possibilities of involvement of the PPAR- $\gamma$ signalling and enhanced Rac1 activity through the HGF/COX-2/ $\mathrm{PGJ}_{2}$ or $\mathrm{PGE}_{2}$ axis, resulting in enhanced efferocytic capability in our experimental setting.

Interestingly, in vivo instillation of apoptotic cells resulted in marked suppression of bleomycin-induced production of proinflammatory mediators, such as TNF- $\alpha$ and MIP-2, inflammatory cell recruitment, and total protein levels in BAL fluid. The increased resolution of inflammation was also confirmed by lung tissue histology showing decreased inflammatory cell infiltration and alveolar structure damage following apoptotic cell instillation. Recent studies have indicated that endogenous and exogenous HGF attenuates inflammatory responses, leukocyte infiltration and inflammatory cytokine gene expression during acute kidney injury and chronic kidney disease $[40,41]$. In addition, HGF reduces allergic airway inflammation [42]. Anti-inflammatory action of HGF has been suggested to be mediated by phosphoinositide 3-kinase/Akt/glycogen synthase kinase- $3 \beta$ and disrupting nuclear factor- $\kappa \mathrm{B}$ signalling [43]. In the present study, co-administration of the neutralising anti-HGF antibody reversed the anti-inflammatory effect mediated by apoptotic Jurkat cell instillation, whereas control IgG was ineffective. Thus, evidence was provided that the reduction of inflammatory mediator production and resolution of inflammation were mediated by sequential induction of HGF following apoptotic cell instillation. Consistent with the in vivo findings, endogenously produced HGF by apoptotic cell treatment plays a role in inhibiting the generation of inflammatory cytokines in macrophages in vitro.

HGF suppresses apoptosis of alveolar epithelial cells in a bleomycin-induced lung fibrosis model [44] and small airway epithelial cells in vitro [45]. Similarly, we found that apoptotic cell instillation reduced apoptosis of alveolar epithelial cells in lung sections 7 days after bleomycin treatment using TUNEL staining and immunoblotting for the cleaved form of caspase-3. Importantly, administration of the neutralising anti-HGF antibody also reversed reduction of caspase- 3 and -9 activities during the early inflammatory phase after bleomycin treatment. These findings emphasise that anti-apoptotic effect of apoptotic cell instillation may be mediated by subsequent HGF induction. The caspase cascade linking to the Fas-FasL pathway or the mitochondrial pathway-mediated apoptosis of alveolar epithelial cells is a pathogenic mechanism of bleomycin-induced lung fibrosis and idiopathic pulmonary fibrosis [44, 46, 47]. Therefore, suppression of alveolar epithelial cell apoptosis by HGF induction following apoptotic cell instillation may 

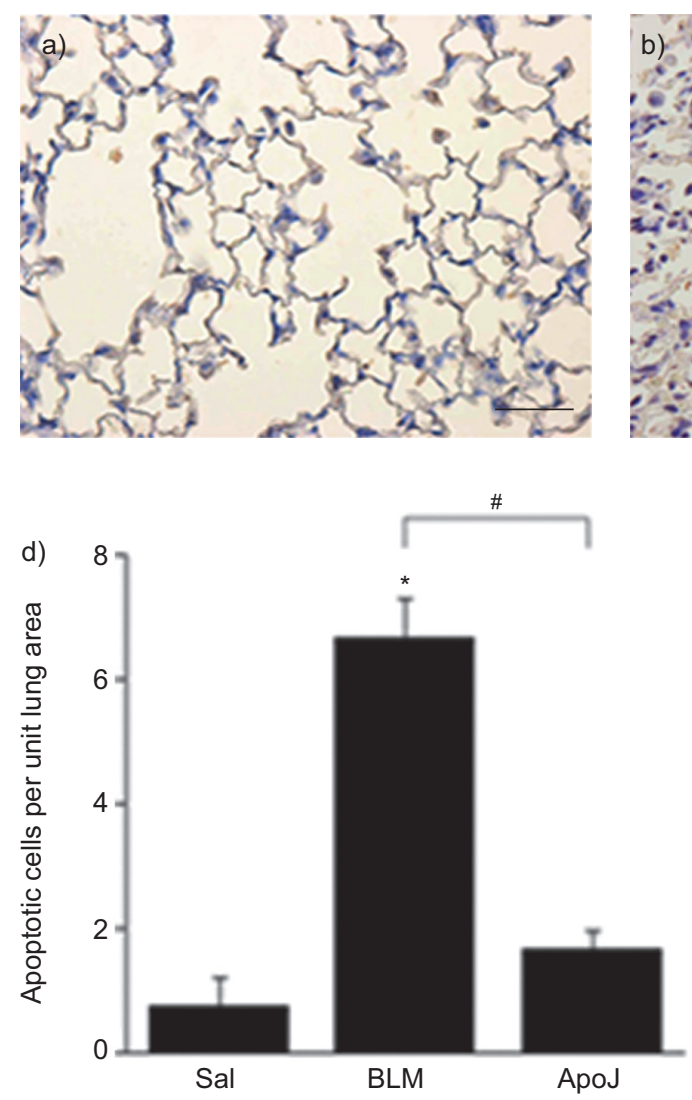
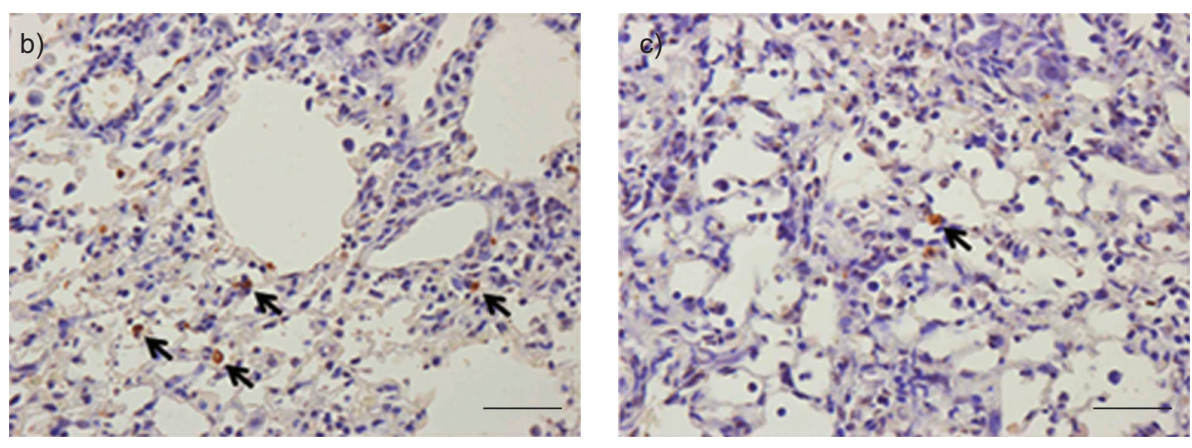

e)

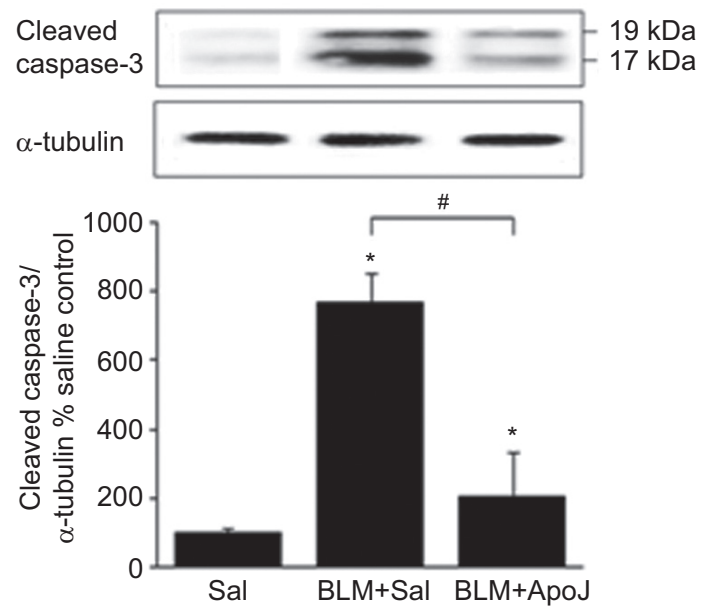

FIGURE 4. Reduction of lung apoptosis by in vivo instillation of apoptotic cells. Mice were instilled intratracheally with saline (Sal) or apoptotic Jurkat T-cells (ApoJ) on day 2 and euthanised on days 3-21 following bleomycin (BLM) treatment. Terminal deoxynucleotidyltransferase-mediated deoxyuridine triphosphate nick end-labelling (TUNEL) stains of lung sections 7 days post-BLM treatment in mice treated with a) Sal, b) BLM+Sal and c) BLM+ApoJ. Scale bars=10 $\mu \mathrm{m}$. Representative results from 10 mice per group are shown. d) The number of TUNEL-positive cells in lung tissues from five mice in each group. e) Lung tissue homogenates were immunoblotted for cleaved caspase-3 fragments. Representative blots and densitometric analysis from five mice per group are shown. Data are presented as relative values compared with saline controls. Data are presented as mean \pm SEM. $n=5$ mice per group. *: $p<0.05$ compared with Sal alone; * : $p<0.05$ for BLM+ApoJ versus BLM+Sal.

lead to reduced prevention of the progressive fibrotic process that follows bleomycin treatment.

Indeed, apoptotic cell instillation reduced the hydroxyproline content in lung tissues, a finding consistent with the anti-fibrotic effect. Reduction of collagen expression was confirmed by analysis of lung histology 21 days after bleomycin treatment. These findings indicate that the persistently enhanced production of HGF following in vivo instillation of apoptotic cells functions in an anti-fibrosis manner. Bleomycin-induced lung injury in mice, concomitant to or after treatment with HGF, repressed fibrotic morphological changes 2 and 4 weeks after bleomycin treatment [48-50]. This reduction in fibrosis was associated with increased bronchial and alveolar epithelial cell proliferation. Indeed, we found that apoptotic cell instillation mimicked this HGF effect by enhancing DNA synthesis for alveolar epithelial cell proliferation (fig. S5).

TGF- $\beta 1$ has been shown to be further enhanced when apoptotic cells were exposed in vitro and in vivo to LPSstimulated macrophages and lungs $[18,24]$. The enhanced TGF- $\beta 1$ promoted resolution of inflammation. In addition to enhanced HGF production, in the present study, we found an upregulation of the production of TGF- $\beta 1$ after apoptotic cell instillation during the early inflammatory phases (days 2 and 3 after bleomycin treatment). Thereafter, the levels of TGF- $\beta 1$ declined to nearly the levels shown in the bleomycin-only group (days 5 and 7). These data suggest that the antiinflammatory role of TGF- $\beta 1$ induced by in vivo instillation of apoptotic cells may be effective during the early phase (days 2 and 3), but minimally effective during the later phases (days 5 and 7). Interestingly, neutralisation of HGF tends to further increase the levels of TGF- $\beta 1$ at 3 days, although it was not statistically significant, but did not affect it at 7 days. Indeed, in vitro treatment of macrophages with apoptotic cells with antiHGFR (c-Met) antibody enhanced TGF- $\beta 1$ production (fig. S6). Collectively, these findings suggest that the enhanced HGF production tends to downregulate the levels of TGF- $\beta 1$ to prevent its overexpression, but it appears to have little effect on basal TGF- $\beta 1$ expression.

Recent studies demonstrate that HGF reduces fibrogenesis by not only inhibiting TGF- $\beta$ signalling but also down-regulating TGF- $\beta$ in several experimental models of fibrosis $[10,15,49,51$, 52]. In the present study, the production of TGF- $\beta 1$ was downregulated after apoptotic cell instillation during the late fibrotic phases (days 14 and 21 after bleomycin), while the HGF production continuously increased and peaked at 21 days, indicating that the antifibrotic action in the BLM+ApoJ group is 

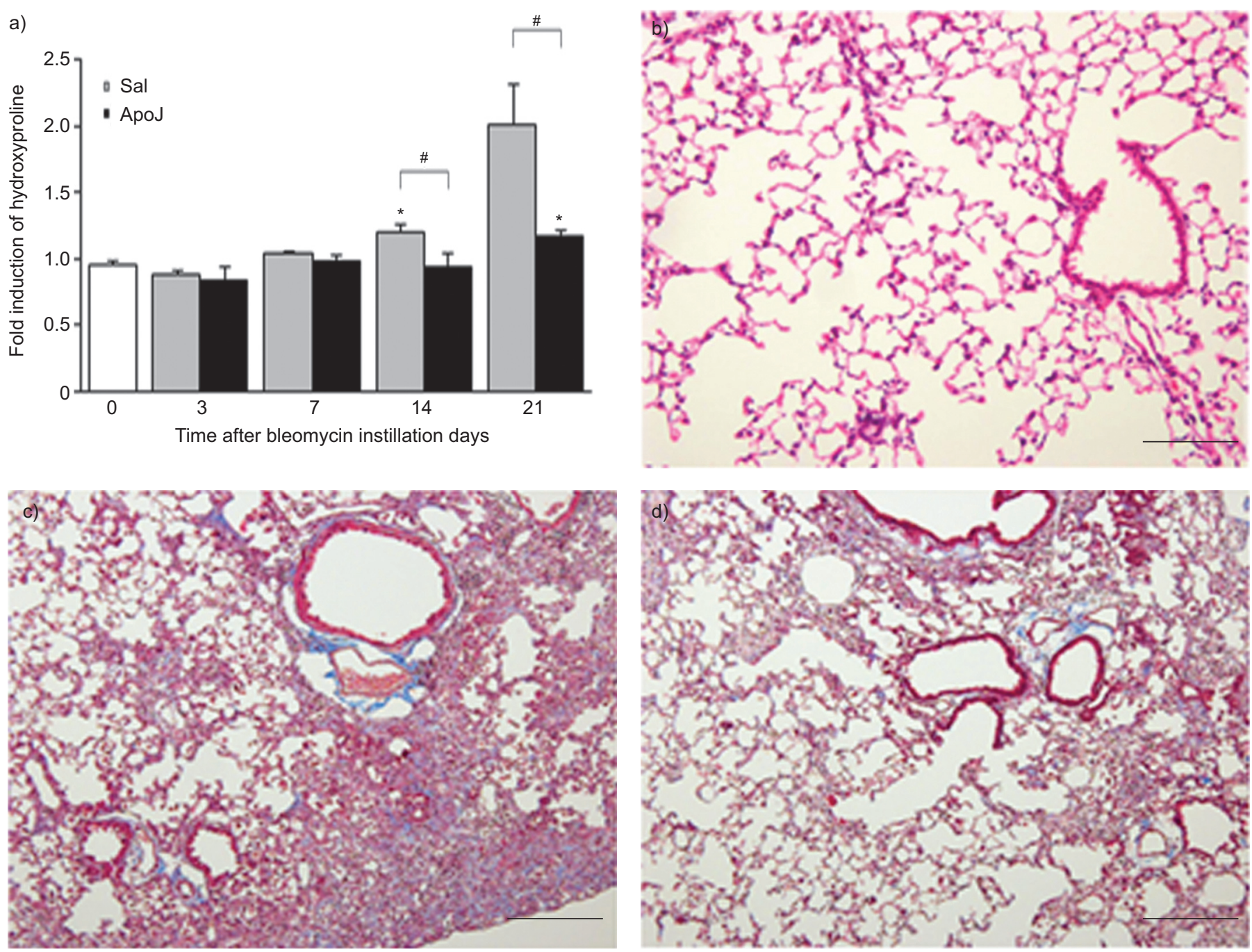

FIGURE 5. Reduction of hydroxyproline in lung tissue by in vivo instillation of apoptotic cells. Mice were instilled intratracheally with saline (Sal) or apoptotic Jurkat Tcells (ApoJ) 2 days after bleomycin (BLM) treatment and lungs were harvested on days 3, 7, 14 and 21. a) Collagen deposition in the whole lung was determined by measuring hydroxyproline content. Data are presented as mean \pm SEM. $n=5$ mice per group. *: $p<0.05$ compared with Sal; \#: $p<0.05$ for BLM+ApoJ versus BLM+Sal. Lung sections were visualised with Masson's trichrome staining. b) Sal; c) BLM+Sal; d) BLM+ApoJ. Scale bars $=10 \mu m$. Representative results from five mice per group are shown.

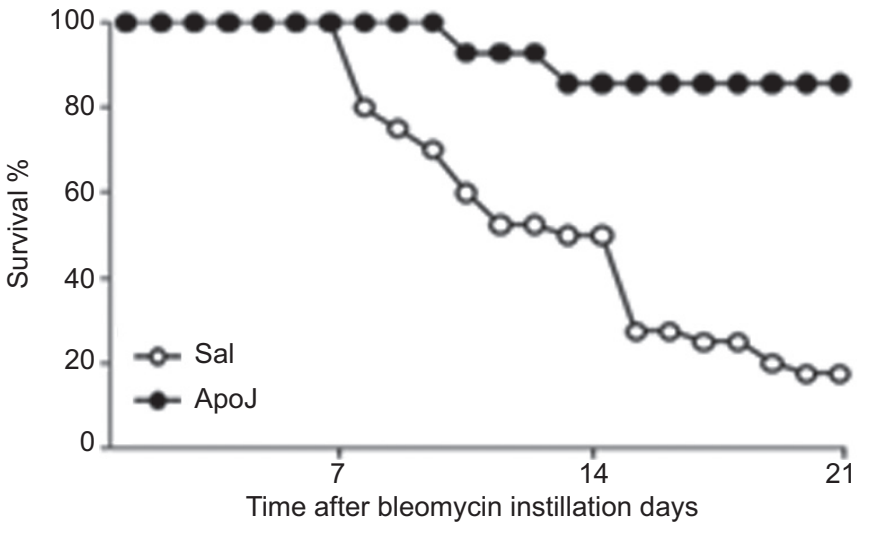

FIGURE 6. Changes in mouse survival rates. Mice were instilled intratracheally with saline (Sal) or apoptotic Jurkat T-cells (ApoJ) 2 days after bleomycin treatment. The percentage of surviving mice was plotted over a 21-day period. $n=20$ mice per group. concordant with an increase in HGF and a decrease in TGF- $\beta 1$ levels as compared with the BLM+Sal group. Whether highly increased HGF downregulates TGF- $\beta 1$ in the fibrotic phases is not clear in our current experimental setting. However, these data are consistent with the notion that the levels or ratio of HGF/TGF- $\beta 1$ may play an important role in the balance of injury and repair during the late fibrotic phases. Accordingly, the net effect of apoptotic cell instillation in the late stage of bleomycin treatment appears to shift balance between HGF and TGF- $\beta 1$, favouring the antifibrotic HGF.

In conclusion, in vivo exposure to apoptotic cells resulted in enhanced HGF mRNA and protein expression above the high HGF basal levels found in bleomycin-stimulated lungs. Persistent increases in HGF production following apoptotic cell instillation may enhance anti-inflammatory, antiapoptotic and antifibrotic effects, resulting in improved alveolar wound repair. Thus, early apoptotic cell instillation specific for lung 
injury lesions may represent considerable implications for a therapeutic strategy for treating pulmonary inflammation and fibrosis.

\section{SUPPORT STATEMENT}

This study was supported by a National Research Foundation grant funded by the Korean government (MEST) (2008-0061522 and 20100029353).

\section{STATEMENT OF INTEREST}

None declared.

\section{REFERENCES}

1 Sakai T, Satoh K, Matsushima K, et al. Hepatocyte growth factor in bronchoalveolar lavage fluids and cells in patients with inflammatory chest diseases of the lower respiratory tract: detection by RIA and in situ hybridization. Am J Respir Cell Mol Biol 1997; 16: 388-397.

2 Geiser T. Idiopathic pulmonary fibrosis-a disorder of alveolar wound repair? Swiss Med Wkly 2003; 133: 405-411.

3 Adamson IY, Young L, Bowden DH. Relationship of alveolar epithelial injury and repair to the induction of pulmonary fibrosis. Am J Pathol 1988; 41: 377-383.

4 Thannickal VJ, Toews GB, White ES, et al. Mechanisms of pulmonary fibrosis. Annu Rev Med 2004; 5: 395-417.

5 Harari S, Caminati A. IPF: new insight on pathogenesis and treatment. Allergy 2010; 5: 537-553.

6 Vandivier RW, Henson PM, Douglas IS. Burying the dead. The impact of failed apoptotic cell removal (efferocytosis) on chronic inflammatory lung disease. Chest 2006; 129: 1673-1682.

7 Morimoto K, Amano H, Sonoda F, et al. Alveolar macrophages that phagocytose apoptotic neutrophils produce hepatocyte growth factor during bacterial pneumonia in mice. Am J Respir Cell Mol Biol 2001; 24: 608-615.

8 Liu Y. Hepatocyte growth factor in kidney fibrosis: therapeutic potential and mechanisms of action. Am J Physiol Renal Physiol 2004; 287: F7-F16.

9 Michalopoulos GK, DeFrances MC. Liver regeneration. Science 1997; 276: 60-66.

10 Atta HM, AI-Hendy A, Salama SA, et al. Low-dose simultaneous delivery of adenovirus encoding hepatocyte growth factor and vascular endothelial growth factor in dogs enhances liver proliferation without systemic growth factor elevation. Liver Int 2009; 29: 1022-1030.

11 Matsumoto K, Nakamura T. Hepatocyte growth factor: renotropic role and potential therapeutics for renal diseases. Kidney Int 2001; 59: 2023-2038.

12 Vargas GA, Hoeflich A, Jehle PM. Hepatocyte growth factor in renal failure: promise and reality. Kidney Int 2000; 57: 1426-1436.

13 Ware LB, Matthay MA. Keratocyte and hepatocyte growth factors in the lung: roles in lung development, inflammation, and repair. Am J Physiol Lung Cell Mol Physiol 2002; 282: L924-L940.

14 Watanabe M, Ebina M, Orson FM, et al. Hepatocyte growth factor gene transfer to alveolar septa for effective suppression of lung fibrosis. Mol Ther 2005; 12: 58-67.

15 Shukla MN, Rose JL, Ray R, et al. Hepatocyte growth factor inhibits epithelial to myofibroblast transition in lung cells via Smad7. Am J Respir Cell Mol Biol 2009; 40: 643-653.

16 Yang J, Dai C, Liu Y. Hepatocyte growth factor suppresses renal interstitial myofibroblast activation and intercepts Smad signal transduction. Am J Pathol 2003; 163: 621-632.

17 Rao GVS, Tinkle S, Weissman DN, et al. Efficacy of a technique for exposing the mouse lung to particles aspirated from the pharynx. J Toxicol Environ Health A 2003; 66: 1441-1452.
18 Huynh ML, Fadok VA, Henson PM. Phosphatidylserine-dependent ingestion of apoptotic cells promotes TGF- $\beta 1$ secretion and the resolution of inflammation. J Clin Invest 2002; 109: 41-50.

19 Moon C, Lee YJ, Park HJ, et al. N-acetylcysteine inhibits RhoA and promotes apoptotic cell clearance during intense lung inflammation. Am J Respir Crit Care Med 2010; 81: 374-387.

20 Wenzel SE, Szefler SJ, Leung DY, et al. Bronchoscopic evaluation of severe asthma: persistent inflammation associated with high dose glucocorticoids. Am J Respir Crit Care Med 1997; 156: 737-743.

21 Hoffman PR, deCathelineau AM, Ogden CA, et al. Phosphatidylserine (PS) induces PS receptor-mediated macropinocytosis and promotes clearance of apoptotic cells. J Cell Biol 2001; 155: 649-659.

22 Hartree EF. Determination of protein: a modification of the Lowry method that gives a linear photometric response. Anal Biochem 1972; 48: 422-427.

23 Park HJ, Choi YH, Cho YJ, et al. RhoA-mediated signaling upregulates hepatocyte growth factor gene and protein expression in response to apoptotic cells. J Leukoc Biol 2011; 89: 309-411.

24 Fadok VA, Bratton DL, Konowal A, et al. Macrophages that have ingested apoptotic cells in vitro inhibit proinflammatory cytokine production through autocrine/paracrine mechanisms involving TGF- $\beta$, PGE $_{2}$, and PAF. J Clin Invest 1998; 101: 890-898.

25 Adamson IYR, Bakowska J. Relationship of keratinocyte growth factor and hepatocyte growth factor levels in rat lung lavage fluid to epithelial cell regeneration after bleomycin. Am J Pathol 1999; 155: 949-954.

26 Lee SL, Huang PY, Roller P, et al. Matriptase/epithin participates in mammary epithelial cell growth and morphogenesis through HGF activation. Mech Dev 2010; 127: 82-95.

27 Sun R, Jaruga B, Kulkarni S, et al. IL-6 modulates hepatocyte proliferation via induction of $\mathrm{HGF} / \mathrm{p} 21^{\mathrm{cip} 1}$ : regulation by SOCS3. Biochem Biophys Res Commun 2005; 338: 1943-1949.

28 Quesnel C, Marchand-Adam S, Fabre A, et al. Regulation of hepatocyte growth factor secretion by fibroblasts in patients with acute lung injury. Am J Physiol Lung Cell Mol Physiol 2008; 294: L334-L343.

29 Gohda E, Takebe T, Sotani T, et al. Induction of hepatocyte growth factor/scatter factor by interferon-gamma in human leukemia cells. J Cell Physiol 1998; 174: 107-114.

30 Zhang A, Wang Y, Ye Z, et al. Mechanism of TNF- $\alpha$-induced migration and hepatocyte growth factor production in human mesenchymal stem cells. J Cell Biochem 2010; 111: 469-475.

31 Nayeri F, Xu J, Abdiu A, et al. Autocrine production of biologically active hepatocyte growth factor (HGF) by injured human skin. J Dermatol Sci 2006; 43: 49-56.

32 Mildner M, Mlitz V, Gruber F, et al. Hepatocyte growth factor establishes autocrine and paracrine feedback loops for the protection of skin cells after UV irradiation. J Invest Dermatol 2007; 27: 2637-2644.

33 Onimaru Y, Tsukasaki K, Murata K, et al. Autocrine and/or paracrine growth of aggressive ATLL cells caused by HGF and c-Met. Intnatl J Oncol 2008; 33: 697-703.

34 Fernandez-Boyanapalli R, Frasch SC, Riches DW, et al. PPAR $\gamma$ activation normalizes resolution of acute sterile inflammation in murine chronic granulomatous disease. Blood 2010; 116: $4512-4522$.

35 Asada K, Sasaki S, Suda T, et al. Antiinflammatory roles of peroxisome proliferator-activated receptor gamma in human alveolar macrophages. Am J Respir Crit Care Med 2004; 169: 195-200.

36 Majai G, Sarang Z, Csomós K, et al. PPAR $\gamma$-dependent regulation of human macrophages in phagocytosis of apoptotic cells. Eur J Immunol 2007; 37: 1343-1354.

37 Nakaya M, Tanaka M, Okabe Y, et al. Opposite effects of rho family GTPases on engulfment of apoptotic cells by macrophages. J Biol Chem 2006; 281: 8836-8842. 
38 Desvergne B, Wahli W. Peroxisome proliferator-activated receptors: nuclear control of metabolism. Endocr Rev 1999; 20: 649-688.

39 Frasch SC, Fernandez-Boyanapalli RF, Berry KZ, et al. Signaling via macrophage G2A enhances efferocytosis of dying neutrophils by augmentation of Rac activity. J Biol Chem 2011; 286: 12108-12122.

40 Homsi E, Janino P, Amano M, et al. Endogenous hepatocyte growth factor attenuates inflammatory response in glycerolinduced acute lung injury. Am J Nephrol 2009; 29: 283-291.

41 Gong R, Rifai A, Dworkin LD. Anti-inflammatory effect of hepatocyte growth factor in chronic kidney disease: targeting the inflamed vascular endothelium. J Am Soc Nephrol 2006; 17: 2464-2473.

42 Ito W, Kanehiro A, Matsumoto K, et al. Hepatocyte growth factor attenuates airway hyperresponsiveness, inflammation, and remodeling. Am J Respir Cell Mol Biol 2005; 32: 268-280.

43 Giannopoulou M, Dai C, Tan X, et al. Hepatocyte growth factor exerts its anti-inflammatory action by disrupting nuclear factor- $\mathrm{\kappa B}$ signaling. Am J Pathol 2008; 173: 30-41.

44 Gazdhar A, Fachinger P, van Leer C, et al. Gene transfer of hepatocyte growth factor by electroporation reduces bleomycininduced lung fibrosis. Am J Physiol Lung Cell Mol Physiol 2007; L529-L536.

45 Okada M, Sugita K, Inukai $\mathrm{T}$, et al. Hepatocyte growth factor protects small airway epithelial cells from apoptosis induced by tumor necrosis factor- $\alpha$ or oxidative stress. Pediatr Res 2004; 56: 336-344.

46 Chen JJ, Sun Y, Nabel GJ. Regulation of the proinflammatory effects of Fas ligand (CD95L). Science 1998; 282: 1714-1717.

47 Kuwano K, Kunitake R, Maeyama $\mathrm{T}$, et al. Attenuation of bleomycin-induced pneumopathy in mice by a caspase inhibitor. Am J Physiol Lung Cell Mol Physiol 2001; 280: L316-L325.

48 Yaekashiwa M, Nakayma S, Ohnuma K, et al. Simultaneous or delayed administration of hepatocyte growth factor equally represses the fibrotic changes in murine lung injury induced by bleomycin. Am J Respir Crit Care Med 1997; 156: 1937-1944.

49 Dohi M, Hasegawa T, Yamamoto K, et al. Hepatocyte growth factor attenuates collagen accumulation in a murine model of pulmonary fibrosis. Am J Respir Crit Care Med 2000; 162: 2302-2307.

50 Lekushi K, Taniyama Y, Azuma J, et al. Hepatocyte growth factor attenuates renal fibrosis through TGF- $\beta 1$ suppression by apoptosis of myofibroblasts. J Hypertens 2010; 28: 2454-2461.

$51 \mathrm{Yu} \mathrm{Y,} \mathrm{Lu} \mathrm{L,} \mathrm{Qian} \mathrm{X,} \mathrm{et} \mathrm{al.} \mathrm{Antifibrotic} \mathrm{effect} \mathrm{of} \mathrm{hepatocyte} \mathrm{growth}$ factor-expressing meshenchymal stem cells in small-for-size liver transplant rats. Stem Cells Dev 2010; 19: 903-914.

52 Xia JL, Dai C, Michalopoulos GK, et al. Hepatocyte growth factor attenuates liver fibrosis induced by bile duct ligation. Am J Pathol 2006; 168: 1500-1512. 